\title{
RADIOCARBON AND DENDROCHRONOLOGICAL DATING OF LOGBOATS FROM POLAND
}

\author{
Anna Pazdur ${ }^{1}$ Marek Krąpiec ${ }^{2}$ Adam Michczyński ${ }^{1}$ Waldemar Ossowski ${ }^{3}$ \\ ABSTRACT. The earliest dating of samples taken from logboats found in the area of Poland was done at the Gliwice Radio- \\ carbon Laboratory in the late 1970s and early 1980s. After a 10-year break, the study of their chronology was renewed. The \\ ${ }^{14} \mathrm{C}$ dates (56) include all previously published and new, unpublished results obtained during last several years. Here, we dis- \\ cuss and provide probabilistic interpretation of the calendar age of the dated boats. The calibration of ${ }^{14} \mathrm{C}$ dates was done with \\ the OxCal program for dates less than $300 \mathrm{BP}$, and with the GdCALIB program for all remaining dates. In distribution of cal- \\ ibrated dates we find a lack of samples between the ages of around $800 \mathrm{BC}$ and $300 \mathrm{AD}$. This result is surprising and differs \\ from results observed for Central Europe. The remaining age ranges, with high frequency of dates, are in good coincidence \\ with similar periods obtained for Central Europe. \\ Tree-ring dating of oak logboats was carried out on 60 growth sequences, dated against standard chronologies defined for the \\ area of Poland. The results of ${ }^{14} \mathrm{C}$ dating and tree-ring analyses give consistent chronologies.
}

\section{INTRODUCTION}

Logboats are a relatively recent source of archaeological information in Poland. It was only when natural methods of absolute dating became widespread that it became possible to establish the age of these boats and to examine their cultural and historical connections. In 1995, the Polish Maritime Museum started to work on a catalog of all the logboats in Poland, which would contain a complete scientific documentation of each one and determine its age on the basis of absolute dating.

Along with rafts, logboats constitute initial forms from which plank boats were built in the earlier Middle Ages. In spite of the fact that more perfect means of water transportation have been developed, logboats are still used for fishing in some places at the Bug River today.

The major obstacle in studies on boats is determining the time and place of their origin. The logboats collected in Polish museums come mainly from opportune discoveries. They were found in water bodies or in their surroundings without any accompanying artifacts that could help to determine the time of their origin by means of archaeological methods. The lack of chronological benchmarks in the surroundings of the boats made it necessary to apply absolute dating methods.

Before World War II, nautical objects from the area of Mazury and Pomerania were dated on the basis of geological and palynological examinations (Bohnsack 1938; Gross 1938). The spread of radiocarbon dating allowed that method to be used to determine the chronology of boatbuilding artifacts in the 1980s (Smolarek 1991; Filipowiak 1988).

\section{MATERIALS}

So far, following a query conducted in Polish museums, it has been possible to determine the number of logboats collected in Polish museums to be about 200, 30 of which are ethnographic artifacts, the builders of which are known. Over and above, we have numerous archival data on opportune discoveries of such craft. At present we can estimate the number of Polish logboats at over 300. Unfortunately, a large number of these are in very poor condition since no measures whatsoever have been

\footnotetext{
${ }^{1}$ Radiocarbon Laboratory, Institute of Physics, Silesian University of Technology, Krzywoustego 2, 44-100 Gliwice, Poland. ${ }^{2}$ Dendrochronological Laboratory, Faculty of Geology, Geophysics and Environmental Protection, University of Mining and Metallurgy, Al. Mickiewicza 30, 30-059 Kraków, Poland

${ }^{3}$ Polish Maritime Museum, Szeroka 67/68, 80-835 Gdańsk, Poland
}

(C) 2001 by the Arizona Board of Regents on behalf of the University of Arizona RADIOCARBON, Vol 43, Nr 2A, 2001, p 403-415

Proceedings of the 17th International ${ }^{14} \mathrm{C}$ Conference, edited by I Carmi and $\mathrm{E}$ Boaretto 
undertaken to preserve them. Also, in many cases, there are no records of the site or circumstances of their discovery (Ossowski 1999).

One hundred and thirty-two logboats have been subjected to ${ }^{14} \mathrm{C}$ dating and dendrochronological studies so far. Most of the logboats on display in museums are from the late Middle Ages or modern times. So although quite a large number of such boats from various parts of Poland have been accumulated in the country's museums, there is not much that can be said about the use of logboats in times earlier than the late Roman period.

In the modern period, logboats became the most common form of small aquatic craft for transportation and fishery. The results of the current research enable one to make comparisons with attempts hitherto made to outline, if only schematically, the development of the logboat in Poland. The historical logboats of known chronology indicate that a diversity of forms and types of logboats existed in various periods, and it is difficult, at the present stage of research, to follow the evolution of the logboat from the earliest times to the present.

\section{${ }^{14} \mathrm{C}$ Dating}

Most samples were collected and submitted in 1994-1998 by W Ossowski (1999) from the Polish Maritime Museum in Gdańsk and, before that, by P Smolarek (1991). Some of the samples were dated at the request of W Filipowiak (1994) from Maritime Museum in Szczecin. A map with logboat sites, numbered 1 to 51, is shown in the Figure 1.

Samples for ${ }^{14} \mathrm{C}$ dating were mostly taken from the edges of boards of well-preserved and identified fragments of logboats. ${ }^{14} \mathrm{C}$ measurements were applied to those boats that were not suitable for dendrochronological dating or had obtained dendroages that were not exact (see Table 1). For a review of the detailed taphonomy of dated logboats see Ossowski (1999). Most of the samples came from museum pieces; some of them were preserved with organic preservatives, not always identified. Other samples were from the edges of boards from logboats remaining at the bottom of lakes. In order to remove preservatives, ground samples were washed three times in Soxhlet column with a mixture of ethanol and benzene ( $1 \mathrm{~L}$ of solution, at the rate of $1: 2)$, in $70^{\circ} \mathrm{C}$, which lasted for about $5 \mathrm{hr}$. Carefully washed with hot distilled water, samples were treated with hot $4 \% \mathrm{HCl}$, washed again and dried. In Table 1 samples purified this way are marked with an asterisk.

The efficiency of the pretreatment described above can be confirmed by the results obtained for the sample Łaźno (nr 15 in Table 1). The first dating was made after standard AAA treatment and gave $2930 \pm 100$ BP, the second date ( $200 \pm 100$ BP) was obtained for the sample washed in Soxhlet column. Repetition of washing did not change the second ${ }^{14} \mathrm{C}$ date.

In case of well-preserved wood, the sample loss after pretreatment was approximately $10 \%$. Afterwards, the samples were charred and combusted. The obtained $\mathrm{CO}_{2}$ was purified according to the standard procedure used in Gliwice Radiocarbon Laboratory (Pazdur and Pazdur 1986). The measurements of ${ }^{14} \mathrm{C}$ concentration were done in gas-proportional counting. Conventional ${ }^{14} \mathrm{C}$ age of the samples was normalized with $\delta^{13} \mathrm{C}=-25 \%$, according to Stuiver and Polach's procedure (1977).

${ }^{14} \mathrm{C}$ dates were calibrated using the Gliwice calibration program GdCALIB (Pazdur and Michczyńska 1989), except for a group of dates with ${ }^{14} \mathrm{C}$ ages less than $300 \mathrm{BP}$, which were calibrated by OxCAL (Ramsey 1995). Figure 1 shows the results of the calibration procedure in shape of the highest probability intervals with confidence $68 \%$ (thin line) and 95\% (thick line) (Pazdur et al. 1999). 


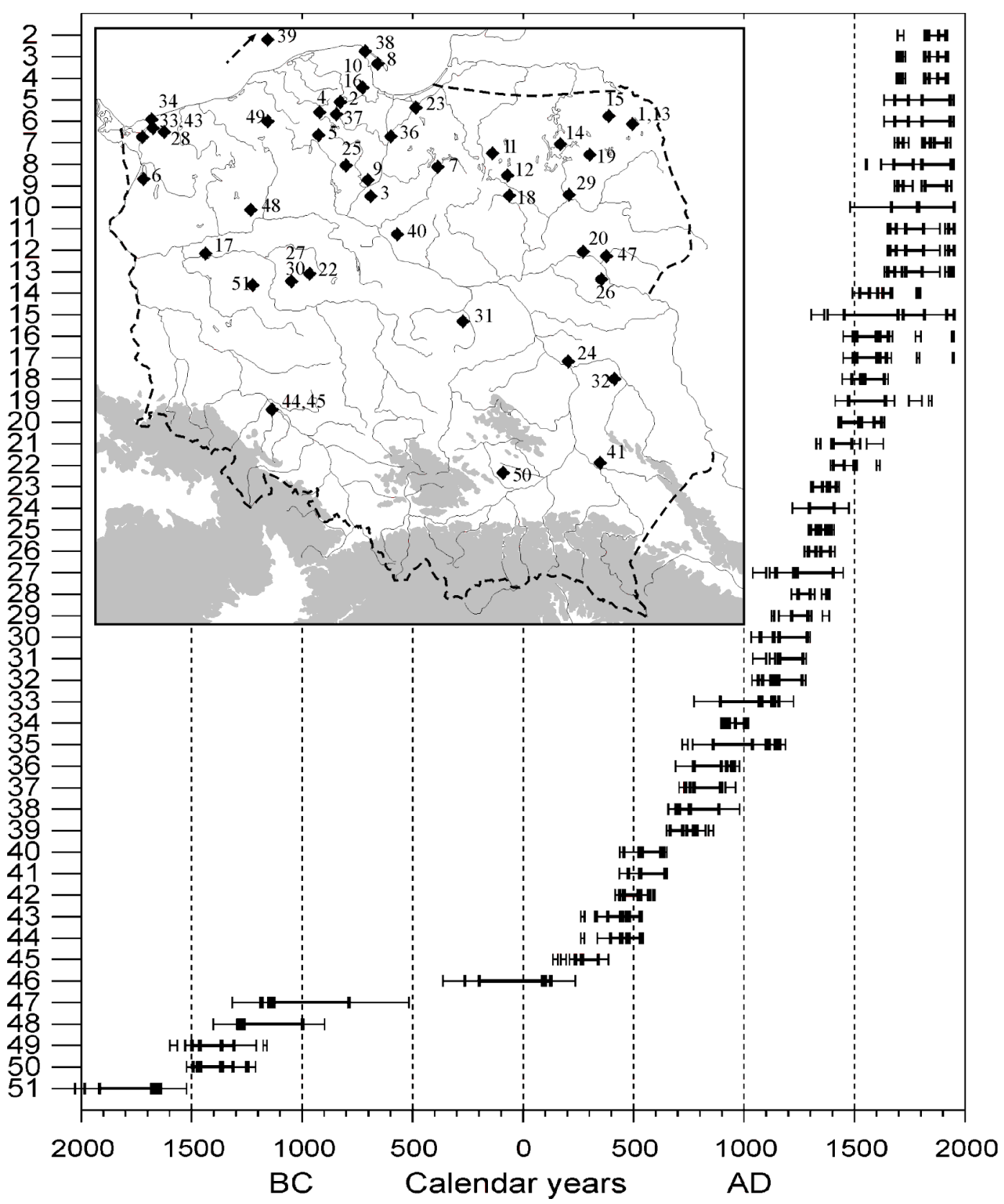

Figure 1 Distribution map of ${ }^{14} \mathrm{C}$ dated logboats and calibration results of ${ }^{14} \mathrm{C}$ dating. The diagram gives the age intervals, within which individual calibrated ages lie, corresponding to the 68\% (thick line) and 95\% (thin line) confidence levels. Only age ranges with the highest probability values are shown. The numbers connected with dates in the left column, changing from 2 to 51, correspond with those on the map (after Pazdur et al. 1999, changed). 
Table 1 Description (name, site, geographical coordinates, species if known, fragment of logboat, location, collector) and conventional and calibrated ages of the samples. Lack of geographical coordinates indicates unknown sites. Age range has been determined with confidence levels $68 \%$ and 95\% using the GdCALIB program, developed in the Gliwice Radiocarbon Laboratory, and the Oxford program OxCal for very young samples. The numbers in brackets mean percent of age range in total probability distribution of calibrated age.

\begin{tabular}{|c|c|c|c|c|}
\hline $\begin{array}{l}\text { Name and sample } \\
\text { description }^{\mathrm{a}}\end{array}$ & Lab nr ${ }^{b}$ & $\begin{array}{c}{ }^{14} \mathrm{C} \\
\text { dendroage }\end{array}$ & $\begin{array}{l}\text { Age range }(68 \%) \\
{[\mathrm{cal} . \mathrm{AD}, \mathrm{BC}]}\end{array}$ & $\begin{array}{l}\text { Age range }(95 \%) \\
{[\text { cal. } \mathrm{AD}, \mathrm{BC}]}\end{array}$ \\
\hline $\begin{array}{l}\text { 1. Wigry-Gawrych } \\
\text { Ruda, Wigry Lake, } \\
\left(54^{\circ} 00^{\prime} \mathrm{N}, 23^{\circ} 01^{\prime} \mathrm{E}\right) \mathrm{Pi} \text { - } \\
\text { nus, EB, UW, Ossowski } \\
\text { W. } 1995\end{array}$ & Gd-7907 & $\begin{array}{c}101.7 \pm 0.8 \\
\mathrm{pMC}\end{array}$ & & \\
\hline $\begin{array}{l}\text { 2. Raduńskie J. } \\
\text { Raduńskie Lake, } \\
\left(54^{\circ} 18^{\prime} \mathrm{N}, 18^{\circ} 00^{\prime} \mathrm{E}\right), P i- \\
\text { nus, EB, PMM, Dyrka M. } \\
1988\end{array}$ & Gd-5482 (Ox) & $<40$ & $\begin{array}{c}1820 \text { AD-1835 AD } \\
(14.7 \%) \\
1882 \text { AD-1914 AD } \\
(53.5 \%)\end{array}$ & $\begin{array}{c}1695 \text { AD-1723 AD } \\
(11.4 \%) \\
1816 \text { AD-1919 AD } \\
(84.0 \%)\end{array}$ \\
\hline $\begin{array}{l}\text { 3. Chełmno } \\
\left(54^{\circ} 20^{\prime} \mathrm{N}, 18^{\circ} 20^{\prime} \mathrm{E}\right) \text {, } \\
\text { wood, EB, PMM, Dyrka } \\
\text { M. } 1988\end{array}$ & Gd-6002 (Ox) & $<50$ & $\begin{array}{l}1701 \text { AD-1717 AD } \\
(9.5 \%) \\
1819 \text { AD-1838 AD } \\
(15.0 \%) \\
1876 \text { AD-1916 AD } \\
(43.7 \%)\end{array}$ & $\begin{array}{c}1690 \text { AD-1731 AD } \\
(17.2 \%) \\
1813 \text { AD-1925 AD } \\
(78.2 \%)\end{array}$ \\
\hline $\begin{array}{l}\text { 4. Mausz J. } \\
\text { Mausz Lake }\left(54^{\circ} 12^{\prime} \mathrm{N},\right. \\
\left.17^{\circ} 42^{\prime} \mathrm{E}\right) \text {, wood, EB, } \\
\text { PMM, Dyrka M. } 1988\end{array}$ & Gd-5483 (Ox) & $<50$ & $\begin{array}{l}1701 \text { AD-1717 AD } \\
(9.5 \%) \\
1819 \text { AD-1838 AD } \\
(15.0 \%) \\
1876 \text { AD-1916 AD } \\
(43.7 \%)\end{array}$ & $\begin{array}{c}1690 \text { AD-1731 AD } \\
(17.2 \%) \\
1813 \text { AD-1925 AD } \\
(78.2 \%)\end{array}$ \\
\hline $\begin{array}{l}\text { 5. Charzykowskie J. } \\
\text { Charzykowskie Lake, } \\
\left(53^{\circ} 47^{\prime} \mathrm{N}, 17^{\circ} 28^{\prime} \mathrm{E}\right), \\
\text { Quercus,EB, UW, Krus- } \\
\text { zelnicki K. } 1978\end{array}$ & Gd-1010 (Ox) & $<150$ & $\begin{array}{c}1683 \text { AD-1744 AD } \\
(21.1 \%) \\
1867 \text { AD-1933 AD } \\
(47.1 \%)\end{array}$ & $\begin{array}{c}1634 \text { AD-1950 AD } \\
(95.4 \%)\end{array}$ \\
\hline $\begin{array}{l}\text { 6. Szczecin-Rubinowy } \\
\text { Staw, Rubinowy Lake, } \\
\left(53^{\circ} 30^{\prime} \mathrm{N}, 14^{\circ} 28^{\prime} \mathrm{E}\right), \\
\text { wood, EB, Museum in } \\
\text { Szczecin, Filipowiak } \\
1984\end{array}$ & Gd-2313 (Ox) & $<150$ & $\begin{array}{l}1683 \text { AD-1744 AD } \\
(21.1 \%) \\
1867 \text { AD-1933 AD } \\
(47.1 \%)\end{array}$ & $\begin{array}{c}1634 \text { AD-1950 AD } \\
(95.4 \%)\end{array}$ \\
\hline $\begin{array}{l}\text { 7. Gim } \\
\text { Gim Lake, } \\
\left(53^{\circ} 34^{\prime} \mathrm{N}, 20^{\circ} 28^{\prime} \mathrm{E}\right), \\
\text { Pinus, EB, Museum in } \\
\text { Olsztyn, Ossowski W. } \\
1995\end{array}$ & Gd-7909 (Ox) & $20 \pm 60 \mathrm{BP}$ & $\begin{aligned} 1699 & \text { AD-1720 AD } \\
& (12.3 \%) \\
1818 & \text { AD-1843 AD } \\
& (16.4 \%) \\
1863 & \text { AD-1917 AD } \\
& (39.5 \%)\end{aligned}$ & $\begin{array}{c}1682 \text { AD-1745 AD } \\
(23.8 \%) \\
1806 \text { AD-1935 AD } \\
(71.6 \%)\end{array}$ \\
\hline $\begin{array}{l}\text { 8. Jastarnia } \\
\left(54^{\circ} 41^{\prime} \mathrm{N}, 18^{\circ} 41^{\prime} \mathrm{E}\right) \text {, } \\
\text { wood, EB, Museum on } \\
\text { Hel, Ossowski } 1997\end{array}$ & Gd-9739 (*,Ox) & $40 \pm 170 \mathrm{BP}$ & $\begin{array}{c}1680 \text { AD-1765 AD } \\
(25.2 \%) \\
1804 \text { AD-1938 AD } \\
(43.0 \%)\end{array}$ & $\begin{array}{c}1512 \text { AD-1597 AD } \\
(6.7 \%) \\
1620 \text { AD-1950 AD } \\
(88.7 \%)\end{array}$ \\
\hline $\begin{array}{l}\text { 9. Świecie } \\
\text { Pinus, Community Cen- } \\
\text { tre in Swiecie, Ossowski } \\
\text { W. } 1998\end{array}$ & Gd-11436 (Ox) & $50 \pm 65 \mathrm{BP}$ & $\begin{array}{c}1697 \text { AD-1721 AD } \\
(12.96 \%) \\
1818 \text { AD-1918 AD } \\
(55.24 \%)\end{array}$ & $\begin{array}{c}1679 \text { AD-1766 AD } \\
(29.57 \%) \\
1804 \text { AD-1938 AD } \\
(65.83 \%)\end{array}$ \\
\hline $\begin{array}{l}\text { 10. Borkowo J. } \\
\text { Głębokie Lake, } \\
\left(54^{\circ} 20^{\prime} \mathrm{N}, 18^{\circ} 20^{\prime} \mathrm{E}\right), P i \text { - } \\
\text { nus, EB, PMM, Dyrkowa } \\
\text { M. } 1981\end{array}$ & Gd-922 (Ox) & $60 \pm 60 \mathrm{BP}$ & $\begin{array}{l}1698 \text { AD-1721 AD } \\
(12.3 \%) \\
1816 \text { AD-1918 AD } \\
(55.9 \%)\end{array}$ & $\begin{array}{l}1680 \text { AD-1764 AD } \\
(28.6 \%) \\
1805 \text { AD-1938 AD } \\
(66.8 \%)\end{array}$ \\
\hline
\end{tabular}


Table 1 Description (name, site, geographical coordinates, species if known, fragment of logboat, location, collector) and conventional and calibrated ages of the samples. Lack of geographical coordinates indicates unknown sites. Age range has been determined with confidence levels $68 \%$ and 95\% using the GdCALIB program, developed in the Gliwice Radiocarbon Laboratory, and the Oxford program OxCal for very young samples. The numbers in brackets mean percent of age range in total probability distribution of calibrated age. (Continued)

\begin{tabular}{|c|c|c|c|c|}
\hline $\begin{array}{l}\text { Name and sample } \\
\text { description }^{\mathrm{a}}\end{array}$ & Lab nr ${ }^{b}$ & $\begin{array}{c}{ }^{14} \mathrm{C} \\
\text { dendroage }\end{array}$ & $\begin{array}{c}\text { Age range }(68 \%) \\
{[\text { cal. } \mathrm{AD}, \mathrm{BC}]}\end{array}$ & $\begin{array}{c}\text { Age range }(95 \%) \\
{[\text { cal. } \mathrm{AD}, \mathrm{BC}]}\end{array}$ \\
\hline $\begin{array}{l}\text { 11. Barczewko } \\
\text { Wadąg River, }\left(53^{\circ} 49^{\prime} \mathrm{N},\right. \\
\left.20^{\circ} 34^{\prime} \mathrm{E}\right), \text { Pinus, EB, Mu- } \\
\text { seum in Olsztyn, Os- } \\
\text { sowski W. } 1995\end{array}$ & Gd-9721 (*,Ox) & $130 \pm 180 \mathrm{BP}$ & $\begin{array}{l}1668 \text { AD-1785 AD } \\
(29.3 \%) \\
1793 \text { AD-1950 AD } \\
(38.9 \%)\end{array}$ & $\begin{array}{c}1480 \text { AD-1950 AD } \\
(95.4 \%)\end{array}$ \\
\hline $\begin{array}{l}\text { 12. Rusek } \\
\left(53^{\circ} 43^{\prime} \mathrm{N}, 23^{\circ} 52^{\prime} \mathrm{E}\right), \mathrm{Pi}- \\
\text { nus, EB, Museum in Szc- } \\
\text { zytno, Ossowski W. } 1995\end{array}$ & Gd-7916 (*,Ox) & $190 \pm 50 \mathrm{BP}$ & $\begin{aligned} & 1660 \text { AD-1688 AD } \\
&(13.6 \%) \\
& 1732 \text { AD-1812 AD } \\
&(40.2 \%) \\
& 1926 \text { AD-1950 AD } \\
&(14.4 \%)\end{aligned}$ & $\begin{array}{c}1649 \text { AD-1887 AD } \\
(78.2 \%) \\
1911 \text { AD-1950 AD } \\
(17.2 \%)\end{array}$ \\
\hline $\begin{array}{l}\text { 13. Wigry-Binduga } \\
\text { Wigry Lake, } \\
\left(54^{\circ} 00^{\prime} \mathrm{N}, 23^{\circ} 00^{\prime} \mathrm{E}\right), P i \text { - } \\
\text { nus, EB, UW, Ossowski } \\
\text { W. } 1995\end{array}$ & Gd-7915 (*,Ox) & $190 \pm 50 \mathrm{BP}$ & $\begin{aligned} 1660 & \text { AD-1688 AD } \\
& (13.6 \%) \\
1732 & \text { AD-1812 AD } \\
& (40.2 \%) \\
1926 & \text { AD-1950 AD } \\
& (14.4 \%)\end{aligned}$ & $\begin{aligned} 1649 & \text { AD-1887 AD } \\
& (78.2 \%) \\
1911 & \text { AD-1950 AD } \\
& (17.2 \%)\end{aligned}$ \\
\hline $\begin{array}{l}\text { 14. Majcz } \\
\text { Majcz Lake, } \\
\left(53^{\circ} 46^{\prime} \mathrm{N}, 21^{\circ} 28^{\prime} \mathrm{E}\right), \\
\text { Quercus, EB, UW, Os- } \\
\text { sowski } 1995\end{array}$ & Gd-7905 (Ox) & $200 \pm 50 \mathrm{BP}$ & $\begin{aligned} 1656 & \text { AD-1686 AD } \\
& (15.7 \%) \\
1736 & \text { AD-1810 AD } \\
& (38.9 \%) \\
1929 & \text { AD-1950 AD } \\
& (13.6 \%)\end{aligned}$ & $\begin{array}{c}1643 \text { AD-1823 AD } \\
(68.7 \%) \\
1830 \text { AD-1886 AD } \\
(8.6 \%) \\
1911 \text { AD-1950 AD } \\
(18.1 \%)\end{array}$ \\
\hline $\begin{array}{l}\text { 15. Łaźno } \\
\text { Łaźno Lake }\left(54^{\circ} 07^{\prime} \mathrm{N},\right. \\
\left.22^{\circ} 13^{\prime} \mathrm{E}\right), \text { Quercus, EB, } \\
\text { Museum in Suwałki, Os- } \\
\text { sowski W. } 1995\end{array}$ & $\begin{array}{l}\text { Gd-11304 } \\
\text { Gd-10869 (*, Ox })\end{array}$ & $2930 \pm 100 \mathrm{BP}$ & $\begin{array}{c}952 \text { BC-948 BC } \\
(0.9 \%) \\
1263 \text { BC-991 BC } \\
(67.5 \%) \\
1646 \text { AD-1704 AD } \\
(16.37 \%) \\
1715 \text { AD-1819 AD } \\
(30.01 \%) \\
1836 \text { AD-1880 AD } \\
(10.23 \%) \\
1915 \text { AD-1950 AD } \\
(11.59 \%)\end{array}$ & $\begin{array}{c}1396 \text { BC-898 BC } \\
(95.1 \%) \\
\\
1492 \text { AD-1498 AD } \\
(0.95 \%) \\
1509 \text { AD-1600 AD } \\
(11.45 \%) \\
1618 \text { AD-1950 AD } \\
(83 \%)\end{array}$ \\
\hline $\begin{array}{l}\text { 16. Borkowo II, } \\
\text { Głębokie Lake, } \\
\left(54^{\circ} 20^{\prime} \mathrm{N}, 18^{\circ} 20^{\prime} \mathrm{E}\right), P i- \\
\text { nus, EB, PMM, Dyrkowa } \\
\text { M. } 1981\end{array}$ & Gd-1424 (*,Ox) & $270 \pm 40 \mathrm{BP}$ & $\begin{array}{l}1785 \text { AD-1793 AD } \\
(4.8 \%) \\
1631 \text { AD-1669 AD } \\
(40.9 \%) \\
1524 \text { AD-1560 AD } \\
(22.5 \%)\end{array}$ & $\begin{aligned} 1768 & \text { AD-1802 AD } \\
& (10.4 \%) \\
1614 & \text { AD-1678 AD } \\
& (46.9 \%) \\
1492 & \text { AD-1604 AD } \\
& (38.2 \%)\end{aligned}$ \\
\hline $\begin{array}{l}\text { 17. Sieraków } \\
\left(52^{\circ} 10^{\prime} \mathrm{N}, 16^{\circ} 04^{\prime} \mathrm{E}\right) \text {, } \\
\text { Quercus, EB, Archeolog- } \\
\text { ical Museum in Poznań, } \\
\text { Ossowski W. } 1996\end{array}$ & Gd-9764 (*,Ox) & $270 \pm 250 \mathrm{BP}$ & $\begin{array}{c}1452 \text { AD-1698 AD } \\
(43.6 \%) \\
1721 \text { AD-1817 AD } \\
(17.0 \%) \\
1918 \text { AD-1950 AD } \\
(7.6 \%)\end{array}$ & $\begin{array}{c}1305 \text { AD-1363 AD } \\
(3.8 \%) \\
1378 \text { AD-1950 AD } \\
(91.6 \%)\end{array}$ \\
\hline $\begin{array}{l}\text { 18. Wielbark } \\
\left(53^{\circ} 24^{\prime} \mathrm{N}, 20^{\circ} 57^{\prime} \mathrm{E}\right) \text {, } \\
\text { Quercus, EB, Museum in } \\
\text { Szczytno, Ossowski W. } \\
1995\end{array}$ & Gd-7918 (*,Ox) & $300 \pm 60 \mathrm{BP}$ & $\begin{array}{c}1509 \text { AD-1600 AD } \\
(45.7 \%) \\
1618 \text { AD-1660 AD } \\
(22.5 \%)\end{array}$ & $\begin{array}{c}1451 \text { AD-1678 AD } \\
(87.8 \%) \\
1771 \text { AD-1803 AD } \\
(5.7 \%) \\
1940 \text { AD-1950 AD } \\
(1.9 \%)\end{array}$ \\
\hline
\end{tabular}


Table 1 Description (name, site, geographical coordinates, species if known, fragment of logboat, location, collector) and conventional and calibrated ages of the samples. Lack of geographical coordinates indicates unknown sites. Age range has been determined with confidence levels $68 \%$ and 95\% using the GdCALIB program, developed in the Gliwice Radiocarbon Laboratory, and the Oxford program OxCal for very young samples. The numbers in brackets mean percent of age range in total probability distribution of calibrated age. (Continued)

\begin{tabular}{|c|c|c|c|c|}
\hline $\begin{array}{l}\text { Name and sample } \\
\text { description }^{\mathrm{a}}\end{array}$ & Lab nr ${ }^{b}$ & $\begin{array}{c}{ }^{14} \mathrm{C} \\
\text { dendroage }\end{array}$ & $\begin{array}{c}\text { Age range }(68 \%) \\
{[\text { cal. } \mathrm{AD}, \mathrm{BC}]}\end{array}$ & $\begin{array}{c}\text { Age range }(95 \%) \\
{[\text { cal. AD, BC] }}\end{array}$ \\
\hline $\begin{array}{l}\text { 19. Bręswałd } \\
\text { Orżołek Lake, }\left(53^{\circ} 51^{\prime} \mathrm{N} \text {, }\right. \\
\left.20^{\circ} 24^{\prime} \mathrm{E}\right), \text { Quercus, EB, } \\
\text { Museum in Olsztyn, Os- } \\
\text { sowski W. } 1995\end{array}$ & Gd-7917(*,Ox) & $\begin{array}{l}310 \pm 60 \mathrm{BP} \\
\text { after } 1367 \\
(-6 /+8) \mathrm{AD}\end{array}$ & $\begin{array}{l}1509 \text { AD-1600 AD } \\
(48.1 \%) \\
1617 \text { AD-1654 AD } \\
(20.1 \%)\end{array}$ & $\begin{array}{c}1449 \text { AD-1674 AD } \\
(90.6 \%) \\
1777 \text { AD-1799 AD } \\
(2.9 \%) \\
1944 \text { AD-1950 AD } \\
(1.9 \%)\end{array}$ \\
\hline $\begin{array}{l}\text { 20. Raźny, Bug River, } \\
\left(52^{\circ} 39^{\prime} \mathrm{N}, 21^{\circ} 43^{\prime} \mathrm{E}\right), \mathrm{Pi}^{-} \\
\text {nus, EB, Museum in Sad- } \\
\text { owno, Ossowski W. } 1997\end{array}$ & Gd-7910 (*) & $340 \pm 60 \mathrm{BP}$ & $\begin{array}{l}1614 \text { AD-1635 AD } \\
(11.4 \%) \\
1494 \text { AD-1604 AD } \\
(57.2 \%)\end{array}$ & $\begin{array}{c}1449 \text { AD-1657 AD } \\
(95.4 \%)\end{array}$ \\
\hline $\begin{array}{l}\text { 21. Orżew } \\
\text { Goryń River, Ukraine, } \\
\left(50^{\circ} 45^{\prime} \mathrm{N}, 26^{\circ} 06^{\prime} \mathrm{E}\right), \\
\text { Quercus, EB, Museum of } \\
\text { Architecture in Lwow, } \\
\text { Ossowski W. } 1997\end{array}$ & Gd-11362 (Ox) & $350 \pm 90 \mathrm{BP}$ & $\begin{array}{c}1473 \text { AD-1641 AD } \\
(68.2 \%)\end{array}$ & $\begin{array}{c}1412 \text { AD-1680 AD } \\
(88.7 \%) \\
1748 \text { AD-1805 AD } \\
(4.8 \%) \\
1937 \text { AD-1950 AD } \\
(1.9 \%)\end{array}$ \\
\hline $\begin{array}{l}\text { 22. Białe J. } \\
\text { Białe Lake } \\
\text { wood, EB, Museum in } \\
\text { Gniezno, Pasiciel S. } 1986\end{array}$ & Gd-2556 & $400 \pm 60 \mathrm{BP}$ & 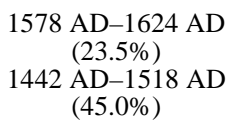 & $\begin{array}{c}1433 \text { AD-1638 AD } \\
(95.4 \%)\end{array}$ \\
\hline $\begin{array}{l}\text { 23. Elbląg } \\
\text { Elblagg River } \\
\left(54^{\circ} 10^{\prime} \mathrm{N}, 19^{\circ} 23^{\prime} \mathrm{E}\right) \\
\text { Quercus, EB, Museum in } \\
\text { Elblagg, Ossowski W. } \\
1995\end{array}$ & Gd-7914 (*) & $460 \pm 60 \mathrm{BP}$ & $\begin{array}{l}1605 \text { AD-1612 AD } \\
(2.9 \%) \\
1407 \text { AD-1489 AD } \\
(65.3 \%)\end{array}$ & $\begin{array}{c}1544 \text { AD-1634 AD } \\
(17.9 \%) \\
1396 \text { AD-1529 AD } \\
(77.4 \%)\end{array}$ \\
\hline $\begin{array}{l}\text { 24. Pawłowice } \\
\text { Vistula River } \\
\left(51^{\circ} 38^{\prime} \mathrm{N}, 21^{\circ} 40^{\prime} \mathrm{E}\right) \text {, } \\
\text { Quercus, EB, Archaeo- } \\
\text { logical Museum in War- } \\
\text { saw, Ossowski W. } 1997\end{array}$ & Gd-7938 & $480 \pm 50 \mathrm{BP}$ & $\begin{array}{c}1409 \text { AD-1458 AD } \\
(68.5 \%)\end{array}$ & $\begin{array}{c}1594 \text { AD-1619 AD } \\
(3.1 \%) \\
1394 \text { AD-1514 AD } \\
(91.0 \%) \\
1324 \text { AD-1337 AD } \\
(1.4 \%)\end{array}$ \\
\hline $\begin{array}{l}\text { 25. Leśno } \\
\left(53^{\circ} 97^{\prime} \mathrm{N}, 17^{\circ} 44^{\prime} \mathrm{E}\right), A l \text { - } \\
\text { nus, UW, Ossowski W. } \\
1998\end{array}$ & Gd-12107 & $550 \pm 60 \mathrm{BP}$ & $\begin{array}{c}1391 \text { AD-1435 AD } \\
(44.11 \%) \\
1315 \text { AD-1347 AD } \\
(24.18 \%)\end{array}$ & $\begin{array}{c}1303 \text { AD-1446 AD } \\
(95.37 \%)\end{array}$ \\
\hline $\begin{array}{l}\text { 26. Wojtkowice } \\
\text { Bug River } \\
\left(52^{\circ} 36^{\prime} \mathrm{N}, 22^{\circ} 25^{\prime} \mathrm{E}\right), \\
\text { Quercus, EB, Museum in } \\
\text { Ciechanowiec, Ossowski } \\
\text { W. } 1996\end{array}$ & Gd-7921 & $570 \pm 50 \mathrm{BP}$ & $\begin{array}{l}1389 \text { AD-1423 AD } \\
(34.5 \%) \\
1313 \text { AD-1350 AD } \\
(33.7 \%)\end{array}$ & $\begin{array}{c}1371 \text { AD-1433 AD } \\
(48.5 \%) \\
1305 \text { AD-1369 AD } \\
(47.0 \%)\end{array}$ \\
\hline $\begin{array}{l}\text { 27. Lednickie J. II } \\
\text { Lednickie Lake } \\
\left(52^{\circ} 31^{\prime} \mathrm{N}, 17^{\circ} 23^{\prime} \mathrm{E}\right) \text {, } \\
\text { Tilia?, EB, Museum in } \\
\text { Lednogóra }\end{array}$ & Lod-272 & $610 \pm 100 \mathrm{BP}$ & $\begin{array}{c}1298 \text { AD-1411 AD } \\
(68.5 \%)\end{array}$ & $\begin{array}{c}1220 \text { AD-1485 AD } \\
(95.5 \%)\end{array}$ \\
\hline $\begin{array}{l}\text { 28. Świeszewo } \\
\left(53^{\circ} 52^{\prime} \mathrm{N}, 15^{\circ} 03^{\prime} \mathrm{E}\right), \\
\text { wood, EB, Museum in } \\
\text { Szczecin, Filipowiak W. } \\
1991\end{array}$ & Gd-5956 & $620 \pm 50 \mathrm{BP}$ & $\begin{aligned} 1377 & \text { AD-1396 AD } \\
& (18.1 \%) \\
1331 & \text { AD-1362 AD } \\
& (29.1 \%) \\
1307 & \text { AD-1329 AD } \\
& (21.0 \%)\end{aligned}$ & $\begin{array}{c}1293 \text { AD-1409 AD } \\
(95.2 \%)\end{array}$ \\
\hline
\end{tabular}


Table 1 Description (name, site, geographical coordinates, species if known, fragment of logboat, location, collector) and conventional and calibrated ages of the samples. Lack of geographical coordinates indicates unknown sites. Age range has been determined with confidence levels $68 \%$ and 95\% using the GdCALIB program, developed in the Gliwice Radiocarbon Laboratory, and the Oxford program OxCal for very young samples. The numbers in brackets mean percent of age range in total probability distribution of calibrated age. (Continued)

\begin{tabular}{|c|c|c|c|c|}
\hline $\begin{array}{l}\text { Name and sample } \\
\text { description }^{\mathrm{a}}\end{array}$ & Lab nr ${ }^{b}$ & $\begin{array}{c}{ }^{14} \mathrm{C} \\
\text { dendroage }\end{array}$ & $\begin{array}{c}\text { Age range }(68 \%) \\
{[\text { cal. } \mathrm{AD}, \mathrm{BC}]}\end{array}$ & $\begin{array}{c}\text { Age range }(95 \%) \\
{[\mathrm{cal} . \mathrm{AD}, \mathrm{BC}]}\end{array}$ \\
\hline $\begin{array}{l}\text { 29. Jurki, Pisa River } \\
\left(53^{\circ} 15^{\prime} \mathrm{N}, 21^{\circ} 52^{\prime} \mathrm{E}\right), \\
\text { Quercus, EB, Heritage } \\
\text { Park in Nowogród, Os- } \\
\text { sowski W. } 1996\end{array}$ & Gd-7922 & $650 \pm 60 \mathrm{BP}$ & $\begin{array}{c}1340 \text { AD-1392 AD } \\
(46.1 \%) \\
1296 \text { AD-1321 AD } \\
(22.0 \%)\end{array}$ & $\begin{array}{c}1279 \text { AD-1412 AD } \\
(95.6 \%)\end{array}$ \\
\hline $\begin{array}{l}\text { 30. Lednickie J. III, } \\
\text { Lednickie Lake, } \\
\left(53^{\circ} 31^{\prime} \mathrm{N}, 17^{\circ} 23^{\prime} \mathrm{E}\right) \text {, } \\
\text { Tilia?, EB, UW, Os- } \\
\text { sowski W. } 1997\end{array}$ & Gd-10625 & $680 \pm 120 \mathrm{BP}$ & $\begin{array}{c}1245 \text { AD-1407 AD } \\
(68.3 \%)\end{array}$ & $\begin{array}{l}1153 \text { AD-1454 AD } \\
(90.2 \%) \\
1115 \text { AD-1145 AD } \\
(1.9 \%) \\
1046 \text { AD-1098 AD } \\
(3.2 \%)\end{array}$ \\
\hline $\begin{array}{l}\text { 31. Sierzchów } \\
\text { Rawka River, }\left(52^{\circ} 08^{\prime} \mathrm{N} \text {, }\right. \\
\left.20^{\circ} 08^{\prime} \mathrm{E}\right), \text { Quercus, EB, } \\
\text { Archaeological Museum } \\
\text { in Warsaw, Ossowski W. } \\
1996\end{array}$ & Gd-7904 & $730 \pm 50 \mathrm{BP}$ & $\begin{array}{c}1368 \text { AD-1371 AD } \\
(2.2 \%) \\
1246 \text { AD-1304 AD } \\
(65.9 \%)\end{array}$ & $\begin{array}{c}1343 \text { AD-1391 AD } \\
(16.1 \%) \\
1220 \text { AD-1318 AD } \\
(79.2 \%)\end{array}$ \\
\hline $\begin{array}{l}\text { 32. Brzeziny, }\left(51^{\circ} 32^{\prime} \mathrm{N} \text {, }\right. \\
\left.22^{\circ} 36^{\prime} \mathrm{E}\right) \text {, Pinus, EB, Mu- } \\
\text { seum of Country in Lub- } \\
\text { lin, Ossowski W. } 1998\end{array}$ & Gd-11437 & $740 \pm 65 \mathrm{BP}$ & $\begin{array}{c}1225 \text { AD-1304 AD } \\
(62.06 \%) \\
1364 \text { AD-1367 AD } \\
(6.34 \%)\end{array}$ & $\begin{array}{c}1172 \text { AD-1323 AD } \\
(76.32 \%) \\
1336 \text { AD-1396 AD } \\
(19.08 \%)\end{array}$ \\
\hline $\begin{array}{l}\text { 33. Kamień Pomorski } \\
\text { Dźwina River, }\left(53^{\circ} 57^{\prime} \mathrm{N} \text {, }\right. \\
\left.14^{\circ} 47^{\prime} \mathrm{E}\right) \text {, wood, EB, Mu- } \\
\text { seum in Szczecin, Filip- } \\
\text { owiak W. } 1984\end{array}$ & Gd-3211 & $770 \pm 60 \mathrm{BP}$ & $\begin{array}{c}1222 \text { AD-1292 AD } \\
(68.0 \%)\end{array}$ & $\begin{array}{c}1348 \text { AD-1390 AD } \\
(4.6 \%) \\
1160 \text { AD-1314 AD } \\
(90.0 \%)\end{array}$ \\
\hline $\begin{array}{l}\text { 34. Wolin-Dziwna, Dzi- } \\
\text { wna River, }\left(53^{\circ} 52^{\prime} \mathrm{N},\right. \\
\left.14^{\circ} 37^{\prime} \mathrm{E}\right) \text {, wood, EB, Mu- } \\
\text { seum in Szczecin, Filip- } \\
\text { owiak W. } 1990\end{array}$ & Gd-6335 A & $810 \pm 80 \mathrm{BP}$ & $\begin{array}{c}1161 \text { AD-1291 AD } \\
(67.6 \%)\end{array}$ & $\begin{array}{c}1031 \text { AD-1305 AD } \\
(94.7 \%)\end{array}$ \\
\hline $\begin{array}{l}\text { 35. Wolin-Dziwna, Dzi- } \\
\text { wna River, }\left(53^{\circ} 52^{\prime} \mathrm{N},\right. \\
\left.14^{\circ} 37^{\prime} \mathrm{E}\right) \text {, wood, EB, Mu- } \\
\text { seum in Szczecin, } \\
\text { Filipowiak W. } 1990\end{array}$ & Gd-6335 B & $835 \pm 55 \mathrm{BP}$ & $\begin{array}{c}1170 \text { AD-1271 AD } \\
(68.4 \%)\end{array}$ & $\begin{array}{l}1153 \text { AD-1286 AD } \\
(78.8 \%) \\
1115 \text { AD-1144 AD } \\
(6.0 \%) \\
1046 \text { AD-1098 AD } \\
(10.5 \%)\end{array}$ \\
\hline 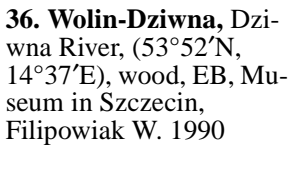 & Gd-6347 C & $850 \pm 70 \mathrm{BP}$ & $\begin{array}{c}1159 \text { AD-1275 AD } \\
(58.7 \%) \\
1126 \text { AD-1134 AD } \\
(3.3 \%) \\
1061 \text { AD-1077 AD } \\
(6.1 \%)\end{array}$ & $\begin{array}{c}1041 \text { AD-1282 AD } \\
(95.2 \%)\end{array}$ \\
\hline $\begin{array}{l}\text { 37. Elbląg II } \\
\text { Quercus, EB, Museum in } \\
\text { Elbląg, Ossowski W. } \\
1995\end{array}$ & Gd-11305 (*) & $1030 \pm 110 \mathrm{BP}$ & $\begin{array}{c}1134 \text { AD-1158 AD } \\
(6.5 \%) \\
1077 \text { AD-1125 AD } \\
(12.8 \%) \\
941 \text { AD-1062 AD } \\
(37.5 \%) \\
894 \text { AD-939 AD } \\
(11.4 \%)\end{array}$ & $\begin{array}{c}780 \text { AD-1241 AD } \\
(95.4 \%)\end{array}$ \\
\hline
\end{tabular}


Table 1 Description (name, site, geographical coordinates, species if known, fragment of logboat, location, collector) and conventional and calibrated ages of the samples. Lack of geographical coordinates indicates unknown sites. Age range has been determined with confidence levels $68 \%$ and 95\% using the GdCALIB program, developed in the Gliwice Radiocarbon Laboratory, and the Oxford program OxCal for very young samples. The numbers in brackets mean percent of age range in total probability distribution of calibrated age. (Continued)

\begin{tabular}{|c|c|c|c|c|}
\hline $\begin{array}{l}\text { Name and sample } \\
\text { description }^{\mathrm{a}}\end{array}$ & Lab nr ${ }^{b}$ & $\begin{array}{c}{ }^{14} \mathrm{C} \\
\text { dendroage }\end{array}$ & $\begin{array}{c}\text { Age range }(68 \%) \\
{[\text { cal. } \mathrm{AD}, \mathrm{BC}]}\end{array}$ & $\begin{array}{c}\text { Age range }(95 \%) \\
{[\text { cal. } \mathrm{AD}, \mathrm{BC}]}\end{array}$ \\
\hline $\begin{array}{l}\text { 38. Nowa Cerkiew } \\
\left(54^{\circ} 11^{\prime} \mathrm{N}, 18^{\circ} 52^{\prime} \mathrm{E}\right), \\
\text { Quercus, EB, PMM, } \\
\text { Dyrkowa } 1984\end{array}$ & Gd-3176 & $\begin{array}{l}1070 \pm 40 \mathrm{BP} \\
\text { after } 959 \mathrm{AD}\end{array}$ & $\begin{array}{c}962 \text { AD-1016 AD } \\
(63.4 \%) \\
899 \text { AD-905 AD } \\
(5.2 \%)\end{array}$ & $\begin{array}{l}941 \text { AD-1022 AD } \\
(75.0 \%) \\
894 \text { AD-937 AD } \\
(20.6 \%)\end{array}$ \\
\hline $\begin{array}{l}\text { 39. Grzybowo } \\
\text { Sominko Lake, }\left(52^{\circ} 08^{\prime} \mathrm{N},\right. \\
\left.20^{\circ} 08^{\prime} \mathrm{E}\right), \text { Quercus, EB, } \\
\text { UW, Ossowski W. } 1997\end{array}$ & Gd-11367 & $\begin{array}{l}1070 \pm 100 \mathrm{BP} \\
\text { after } 1040 \mathrm{AD}\end{array}$ & $\begin{array}{c}1142 \text { AD-1154 AD } \\
(2.4 \%) \\
1091 \text { AD-1117 AD } \\
(5.0 \%) \\
866 \text { AD-1048 AD } \\
(60.3 \%)\end{array}$ & $\begin{array}{c}771 \text { AD-1208 AD } \\
(94.6 \%)\end{array}$ \\
\hline $\begin{array}{l}\text { 40. Puck, Puck Bay, } \\
\left(54^{\circ} 08^{\prime} \mathrm{N}, 20^{\circ} 08^{\prime} \mathrm{E}\right) \text {, } \\
\text { Quercus, EB, Museum in } \\
\text { Puck, Stępień W. } 1980\end{array}$ & Gd-981 & $1190 \pm 70 \mathrm{BP}$ & $\begin{array}{c}911 \text { AD-957 AD } \\
(16.3 \%) \\
778 \text { AD-896 AD } \\
(52.0 \%)\end{array}$ & $\begin{array}{c}689 \text { AD_984 AD } \\
(95.3 \%)\end{array}$ \\
\hline $\begin{array}{l}\text { 41. Gotlandzka Głębia } \\
\left(56^{\circ} 27^{\prime} \mathrm{N}, 19^{\circ} 25^{\prime} \mathrm{E}\right), \\
\text { Quercus, EB, PMM, } \\
\text { Dyrkowa M. } 1984\end{array}$ & Gd-1896 & $\begin{array}{l}1200 \pm 50 \mathrm{BP} \\
\text { after } 730 \mathrm{AD}\end{array}$ & $\begin{array}{c}937 \text { AD-941 AD } \\
(1.5 \%) \\
776 \text { AD-893 AD } \\
(66.9 \%)\end{array}$ & $\begin{array}{c}753 \text { AD-968 AD } \\
(88.3 \%) \\
706 \text { AD-748 AD } \\
(7.3 \%)\end{array}$ \\
\hline $\begin{array}{l}\text { 42. Steklin } \\
\text { Steklin Lake, }\left(52^{\circ} 57^{\prime} \mathrm{N},\right. \\
\left.19^{\circ} 00^{\prime} \mathrm{E}\right), \text { Quercus, EB, } \\
\text { UW, Ossowski } 1995\end{array}$ & Gd-11303 & $1230 \pm 90 \mathrm{BP}$ & $\begin{array}{c}697 \text { AD-887 AD } \\
(68.4 \%)\end{array}$ & $\begin{array}{c}662 \text { AD_980 AD } \\
(95.2 \%)\end{array}$ \\
\hline $\begin{array}{l}\text { 43. Ulanów, San River, } \\
\left(50^{\circ} 34^{\prime} N, 22^{\circ} 04^{\prime} E\right) \text {, } \\
\text { Quercus, EB, PMM } \\
\text { Dyrkowa M. } 1983\end{array}$ & Gd-2064 & $\begin{array}{l}1300 \pm 50 \mathrm{BP} \\
\text { after } 728 \mathrm{AD}\end{array}$ & $\begin{array}{l}731 \text { AD-772 AD } \\
(29.9 \% 0 \\
676 \text { AD-728 AD } \\
(38.4 \% 0\end{array}$ & $\begin{array}{c}836 \text { AD-863 AD } \\
(4.5 \%) \\
657 \text { AD-825 AD } \\
(90.7 \%)\end{array}$ \\
\hline $\begin{array}{l}\text { 44. СMМ/OT/162 } \\
\text { Quercus, EB, PMM, } \\
\text { Dyrkowa M. } 1984\end{array}$ & Gd-1895 & $1490 \pm 50 \mathrm{BP}$ & $\begin{array}{c}547 \text { AD-631 AD } \\
(68.1 \%)\end{array}$ & $\begin{array}{c}496 \text { AD-655 AD } \\
(88.9 \%) \\
450 \text { AD-486 AD } \\
(6.6 \%)\end{array}$ \\
\hline $\begin{array}{l}\text { 45. Kamień Pomorski- } \\
\text { katedra, Quercus, EB, } \\
\text { Cathedral Museum in } \\
\text { Kamień Pomorski, Filip- } \\
\text { owiak W. } 1984\end{array}$ & Gd-2309 & $1490 \pm 60 \mathrm{BP}$ & $\begin{array}{c}540 \text { AD-643 AD } \\
(68.3 \%)\end{array}$ & $\begin{array}{c}446 \text { AD-656 AD } \\
(95.1 \%)\end{array}$ \\
\hline $\begin{array}{l}\text { 46. Wolin Miasto } \\
\text { wood, EB, Museum in } \\
\text { Szczecin, Filipowiak W. } \\
1984\end{array}$ & Gd-2300 & $1570 \pm 40 \mathrm{BP}$ & $\begin{array}{c}447 \text { AD-540 AD } \\
(68.2 \%)\end{array}$ & $\begin{array}{c}573 \text { AD-593 AD } \\
(4.8 \%) \\
422 \text { AD-568 AD } \\
(90.5 \%)\end{array}$ \\
\hline $\begin{array}{l}\text { 47. Kamień Pomorski } \\
\text { from boat, wood, EB, Ca- } \\
\text { thedral Museum in Ka- } \\
\text { mień Pomorski, } \\
\text { Filipowiak W. } 1985\end{array}$ & Gd-1876 & $1630 \pm 50 \mathrm{BP}$ & $\begin{array}{l}476 \text { AD-531 AD } \\
(27.6 \%) \\
397 \text { AD-462 AD } \\
(40.4 \%)\end{array}$ & $\begin{array}{c}330 \text { AD-551 AD } \\
(94.0 \%) \\
265 \text { AD-280 AD } \\
(1.6 \%)\end{array}$ \\
\hline $\begin{array}{l}\text { 48. Lewin Brzeski I } \\
\left(50^{\circ} 45^{\prime} \mathrm{N}, 17^{\circ} 37^{\prime} \mathrm{E}\right) \text {, } \\
\text { Quercus, Museum of } \\
\text { Opole Silesia in Opole }\end{array}$ & Gd-5958 & $\begin{array}{c}1620 \pm 50 \mathrm{BP} \\
\text { late autumn } \\
\text { or winter } \\
371 / 372 \mathrm{AD}\end{array}$ & $\begin{array}{l}473 \text { AD-532 AD } \\
(33.6 \%) \\
411 \text { AD-465 AD } \\
(34.9 \%)\end{array}$ & $\begin{array}{c}336 \text { AD-561 AD } \\
(94.9 \%)\end{array}$ \\
\hline
\end{tabular}


Table 1 Description (name, site, geographical coordinates, species if known, fragment of logboat, location, collector) and conventional and calibrated ages of the samples. Lack of geographical coordinates indicates unknown sites. Age range has been determined with confidence levels $68 \%$ and 95\% using the GdCALIB program, developed in the Gliwice Radiocarbon Laboratory, and the Oxford program OxCal for very young samples. The numbers in brackets mean percent of age range in total probability distribution of calibrated age. (Continued)

\begin{tabular}{|c|c|c|c|c|}
\hline $\begin{array}{l}\text { Name and sample } \\
\text { description }^{\mathrm{a}}\end{array}$ & $\mathrm{Lab} n r^{\mathrm{b}}$ & $\begin{array}{c}{ }^{14} \mathrm{C} \\
\text { dendroage }\end{array}$ & $\begin{array}{l}\text { Age range }(68 \%) \\
{[\text { cal. } \mathrm{AD}, \mathrm{BC}]}\end{array}$ & $\begin{array}{l}\text { Age range }(95 \%) \\
{[\text { cal. AD, BC] }}\end{array}$ \\
\hline $\begin{array}{l}\text { 49. Lewin Brzeski II } \\
\left(50^{\circ} 45^{\prime} \mathrm{N}, 17^{\circ} 37^{\prime} \mathrm{E}\right), \\
\text { Quercus, Museum of } \\
\text { Opole Silesia in Opole }\end{array}$ & Gd-7279 & $\begin{array}{c}1760 \pm 40 \mathrm{BP} \\
\text { ca } 418 \\
(-6 /+9) \mathrm{AD}\end{array}$ & $\begin{array}{l}241 \mathrm{AD}-338 \mathrm{AD} \\
(68.3 \% 0\end{array}$ & $\begin{array}{l}209 \mathrm{AD}-402 \mathrm{AD} \\
(94.2 \%) \\
149 \mathrm{AD}-160 \mathrm{AD} \\
(1.0 \%)\end{array}$ \\
\hline $\begin{array}{l}\text { 50. Kraków Dkra, } \\
\text { Quercus, EB, Archaeo- } \\
\text { logical Museum in Cra- } \\
\text { cow, Krąpiec M. } 1998\end{array}$ & Gd-10773 (*) & $2030 \pm 130 \mathrm{BP}$ & $191 \underset{(68.4 \%)}{\mathrm{BC}-122 \mathrm{AD}}$ & $\begin{array}{c}371 \mathrm{BC}-242 \mathrm{AD} \\
(95.4 \%)\end{array}$ \\
\hline $\begin{array}{l}\text { 51. Śmiełów } \\
\left(53^{\circ} 04^{\prime} \mathrm{N}, 16^{\circ} 28^{\prime} \mathrm{E}\right), \\
\text { Ouercus, EB, Museum in } \\
\text { Smiełów, Ossowski W. } \\
1997\end{array}$ & Gd-10684 (*) & $2750 \pm 150 \mathrm{BP}$ & $\begin{array}{c}1130 \mathrm{BC}-778 \mathrm{BC} \\
(67.9 \%)\end{array}$ & $\begin{array}{c}1313 \text { BC-511 BC } \\
(94.7 \%)\end{array}$ \\
\hline $\begin{array}{l}\text { 52. Laźno } \\
\left(54^{\circ} 07^{\prime} \mathrm{N}, 22^{\circ} 13^{\prime} \mathrm{E}\right) \\
\text { Quercus, EB, Museum in } \\
\text { Suwałki, Ossowski W. } \\
1995\end{array}$ & Gd-11304 (*) & $2930 \pm 100 \mathrm{BP}$ & $\begin{array}{c}952 \text { BC-948 BC } \\
(0.9 \%) \\
1263 \text { BC-991 BC } \\
(67.5 \% 0\end{array}$ & $\begin{array}{c}1396 \mathrm{BC}-898 \mathrm{BC} \\
(95.1 \%)\end{array}$ \\
\hline $\begin{array}{l}\text { 53. Chwalimki } \\
\left(53^{\circ} 45^{\prime} \mathrm{N}, 16^{\circ} 30^{\prime} \mathrm{E}\right), A l- \\
\text { nus, EB, PMM, Ossowski } \\
1997\end{array}$ & Gd-10625 (*) & $3130 \pm 80 \mathrm{BP}$ & $\begin{array}{c}1279 \mathrm{BC}-1269 \mathrm{BC} \\
(3.0 \%) \\
1458 \mathrm{BC}-1298 \mathrm{BC} \\
(59.8 \%) \\
1500 \begin{array}{l}\mathrm{BC}-1480 \mathrm{BC} \\
(5.9 \%)\end{array}\end{array}$ & $\begin{array}{c}1527 \text { BC-1156 BC } \\
(94.0 \%)\end{array}$ \\
\hline $\begin{array}{l}\text { 54. Pinczów } \\
\left(53^{\circ} 45^{\prime} \mathrm{N}, 16^{\circ} 30^{\prime} \mathrm{E}\right) \text {, } \\
\text { Quercus, EB, PMM, Os- } \\
\text { sowski } 1995\end{array}$ & Gd-11304 & $\begin{array}{l}3130 \pm 70 \mathrm{BP} \\
\text { after } 1220 \mathrm{BC}\end{array}$ & $\begin{array}{rl}1272 \mathrm{BC}-1270 \mathrm{BC} & (0.9 \%) \\
1452 & \mathrm{BC}-1303 \mathrm{BC} \\
(63.5 \%) \\
1496 \mathrm{BC}-1484 \mathrm{BC} \\
(3.8 \% 0\end{array}$ & $\begin{array}{c}1249 \mathrm{BC}-1203 \mathrm{BC} \\
(4.2 \%) \\
1522 \mathrm{BC}-1251 \mathrm{BC} \\
(90.9 \%)\end{array}$ \\
\hline $\begin{array}{l}\text { 55. Cieśle } \\
\left(52^{\circ} 22^{\prime} \mathrm{N}, 16^{\circ} 37^{\prime} \mathrm{E}\right) \text {, } \\
\text { Quercus, Archaeological } \\
\text { Museum in Poznań, EB, } \\
\text { Krąpiec M. } 1992\end{array}$ & Gd-6640 & $\begin{array}{c}3470 \pm 100 \mathrm{BP} \\
\text { after } 1700 \\
(-7 /+8) \mathrm{BC}\end{array}$ & $\begin{array}{c}1657 \text { BC-1643 BC } \\
(2.8 \%) \\
1905 \text { BC-1672 BC } \\
(65.3 \% 0\end{array}$ & $\begin{array}{rl}1986 & \mathrm{BC}-1522 \mathrm{BC} \\
& (93.4 \%) \\
2027 & \mathrm{BC}-1996 \mathrm{BC} \\
& (2.0 \%)\end{array}$ \\
\hline
\end{tabular}

\section{DENDROCHRONOLOGY}

Most of the analyzed logboats were made from oak trunks (Quercus robur/petraea). Tree-ring analyses were carried out on 60 oak logboats, selected by researchers from the Polish Maritime Museum (PMM) in Gdańsk (Krapiec and Zielski 1999). In most cases, samples of wood for analyses were taken with an increment borer, suitable for dry wood. Only in cases of badly preserved logboats were slices taken, about $2-3 \mathrm{~cm}$ thick.

Growth sequences of oak logboats were dendrochronologically dated against standard chronologies defined for the area of Poland. These are, among others: southern Poland standard chronologies based on subfossil oaks from alluvial sediments covering 1748-1118 BC, 1018-620 BC, and 474 


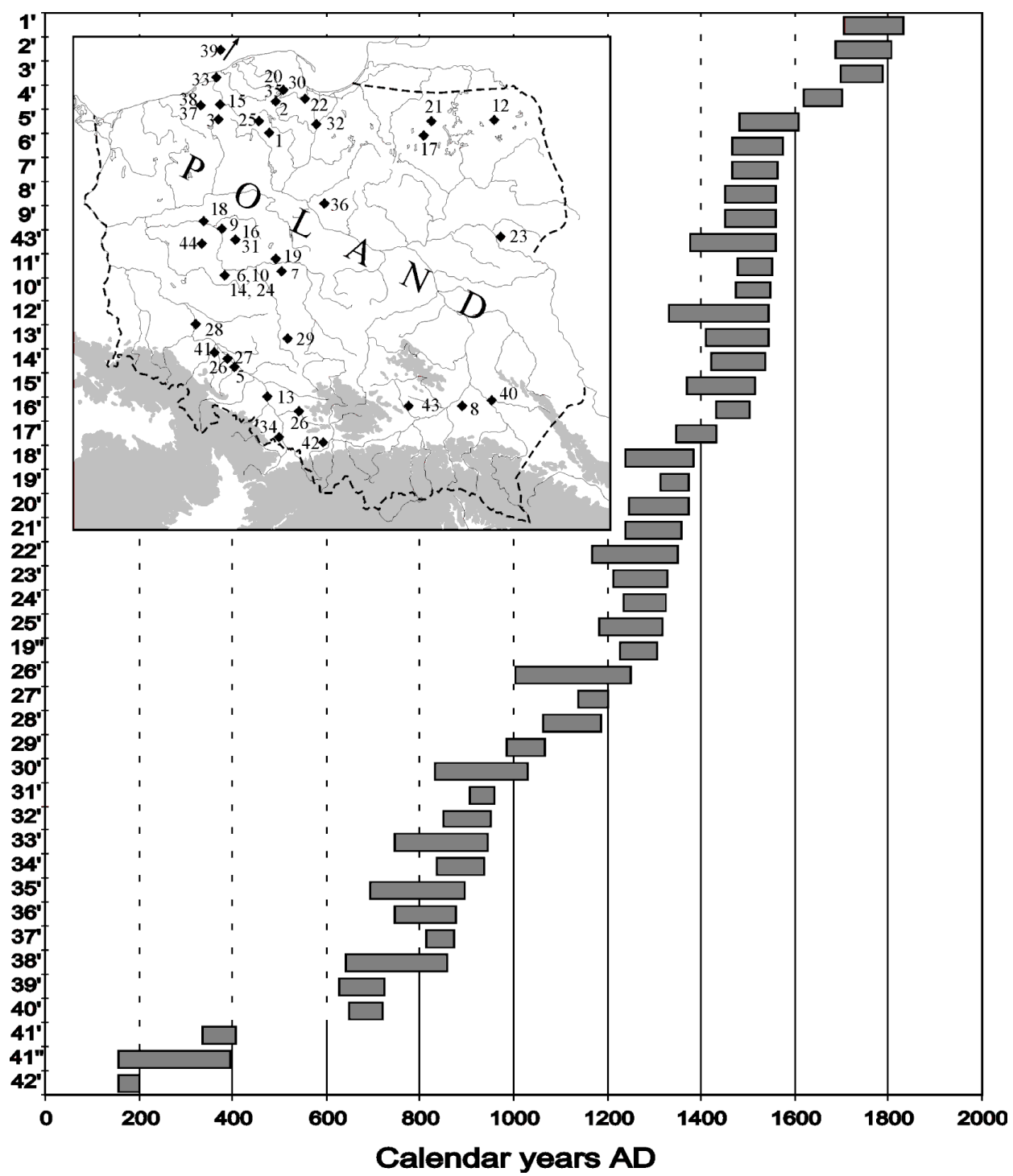

Figure 2 Results of absolute dendrochronological dating of annual growth sequences of oak logboats, with calendar ages from the last 2000 year period, together with their locations. The numbers connected with dating results in the left column correspond with numbers of sites on the map.

BC-1555 AD (Krapiec 1998), regional chronologies for Małopolska (910-1997 AD), Lower Silesia (780-1997 AD) and Wielkopolska (449-1994 AD), based on archaeological and historical wood (Krapiec 1998), as well as chronologies worked out for areas near Pułtusk, Szczecin, Słupsk, and Olsztyn (M Krapiec), Gdańsk Pomerania chronology constructed by T Ważny (1990), and so-called Baltic Wood Chronologies, compiled in western Europe; in England (BALTIC1, BALTIC2; Hillam and Tyers 1995), and in Holland (Bauch and Eckstein 1970). The results of dating of individual objects, together their locations are presented in Figure 2 above. 




Figure 3 The cumulative probability distribution of calibrated ${ }^{14} \mathrm{C}$ ages of 37 logboat samples (straight line) for which the conventional ${ }^{14} \mathrm{C}$ age is less than $300 \mathrm{BP}$. The comparison with the histogram of the dendrochronological dates is shown. Cumulative probability distribution of logboat calibrated ages was divided into phases corresponding to different cultures.

Forty-six logboats were absolutely dated. It turned out that tree-ring dating of logboats, which practically meant dating of individual trees, was highly successful. This was due to the broad comparative material, in the form of regional and local chronologies, as well as to the fact that for the construction of boats, the largest specimens of oaks, dominant in local populations and representative for regional growth pattern, had been selected. 
The oldest of the dendrochronologically dated oak logboats from the area of Poland date back to the Bronze Age. These are one-trunk boats from Cieśla near Buk, dated to around $1700 \mathrm{BC}$ and a boat from Pińczów, made of oak felled around 1220-1215 BC. Logboats from the Roman period are also rare. They are represented by finds from Wolica near Cracow (dated to after 209 AD) and Lewin Brzeski (371 AD and about $418 \mathrm{AD})$.

\section{Frequency Distribution of Calendar Ages}

Figure 3 (on preceding page) presents the cumulative probability distribution obtained by summarizing the probability distributions of calibrated ${ }^{14} \mathrm{C}$ ages of all dated samples with conventional ${ }^{14} \mathrm{C}$ ages higher than 300 BP. We note that probability distribution contains four wide but clearly separated maxima, which correspond to four groups of samples. These groups represent the following age intervals: Bronze Age, Roman period, early Middle Ages, and Middle Ages, and present times. Figure 3 also shows the cumulative probability distributions of calibrated ${ }^{14} \mathrm{C}$ ages for each of group mentioned above.

Probability distribution of all calibrated ${ }^{14} \mathrm{C}$ ages in Figure 3 is compared to the histogram of a number of dendrochronological dates obtained for oak logboats samples in 50-year intervals belonging to periods from the Bronze Age to modern times. Logboats from younger periods, i.e. Middle Ages and Modern Times, are predominant. The frequency distribution pattern of occurrence of logboats from these periods has maxima in the 10th, 14th, and 16th centuries, in agreement with the distribution pattern of ${ }^{14} \mathrm{C}$ dating.

\section{CONCLUSION}

Logboats are important historical artifacts that tell us a great deal about ancient navigation. Until the Middle Ages, logboats are the only survivors of the numerous craft that must have plied Poland's waterways. Research has shown that these boats played a significant part in inland water transport from the Middle Ages to the 19th century. Moreover, not only do logboats provide information about former boat-building techniques, analysis of the use and operating conditions of these craft can tell us a lot about the extent to which water areas were used in former times.

The intervals of ${ }^{14} \mathrm{C}$ calibrated ages, inside which we can observe the maximums of cumulative probability distribution, are consistent with archaeological data. The oldest maximum represents the Bronze Age, when logboats were produced in all of Central Europe. For the time interval from the 3rd to the 17 th century AD, where the cumulative probability distribution has a large maxima, we can observe a very distinct minimum at around 700 AD. This minimum correlates very well with the period of Roman influence, which characterized itself by a lack of logboats among archaeological findings. The other distinct minimum in this part of the cumulative probability distribution can be found around 1100-1200 AD and is followed a large maximum. It allocates well the beginning of the wood floating period.

${ }^{14} \mathrm{C}$ and dendrochronological dating of logboats is consistent and useful for reconstruction of an absolute time scale for archaeological evidence and paleoenvironmental studies.

\section{ACKNOWLEDGMENTS}

The preparation of ${ }^{14} \mathrm{C}$ dating results was financially supported by grants No. 6 P04E 00717 from the Committee for Scientific Research, BW/RMF1/2000 from the Silesian University of Technology, and No. 11.11.140.51 from University of Mining and Metallurgy. 


\section{REFERENCES}

Bauch J, Eckstein D. 1970. Dendrochronological dating of oak panels of Dutch seventeenth-century paintings. Studies of Conservation 15:45-50.

Bohnsack D. 1938. Neue Bondenfunde. Altpreussen 3(1):24-9, 58-62.

Gross H. 1938. Pollenanalytische Untersuchung von 3 Teilprofilen und einigen orgeschichtsfunden von Gohra-Worle (Pommerellen). Blätter für deutsche Vorgeschichte $\mathrm{H} 12: 25-39$.

Filipowiak W. 1988. Początki żeglugi słowiańskiej u ujś cia Odry (The beginning of Slavonic navigation at the Mouth of the River Odra). In: Studia nad etnogeneza Stowian i kultur Europy wczesnośredniowiecznej. Vol. II. p 29-46.

Filipowiak W. 1994. Shipbuilding at the Mouth of the River Odra. In: Westerdahl C, editor. Crossroads in ancient shipbuilding. Oxford: Oxbow Monograph 40. p 83-96.

Hillam J, Tyers I. 1995. Reliability and repeatability in dendrochronological analysis: test using the Flechter archive of panel-painting data. Archaeometry 37(2): 395-405.

Krapiec M. 1998. Oak dendrochronology of the Neoholocene in Poland. Folia Quaternaria 69:5-134.

Krąpiec M, Zielski A. 1999. Dendrochronologiczne datowanie łodzi jednopiennych z obszaru Polski (Dendrochronological dating of logboats from Poland). In: Ossowski W, editor. Studia nad todziami jednopiennymi z obszaru Polski (Study on logboats from Poland). Gdańsk: Marpress. p 5-236.

Ossowski W. 1999. Zróżnicowanie typologiczne i regionalne łodzi jednopiennych $\mathrm{z}$ obszaru Polski
(Taphonomy and regional differences of logboats from Poland) In: Ossowski W, editor. Studia nad todziami jednopiennymi z obszaru Polski (Study on logboats from Poland). Gdańsk: Marpress. p 67-162.

Pazdur A, Goslar T, Michczyński A. 1999. Wiek łodzi jednopiennych $\mathrm{w}$ świetle datowań radiowęglowych (Age of logboats in the light of radiocarbon dating). In: Ossowski W, editor. Studia nad todziami jednopiennymi z obszaru Polski (Study on logboats from Poland). Gdańsk: Marpress. p 259-68.

Pazdur MF, Michczyńska DJ. 1989. Improvement of the procedure for probabilistic calibration of radiocarbon dates. Radiocarbon 31(3):824-32.

Pazdur A, Pazdur MF. 1986 Aparatura pomiarowa Laboratorium C-14 w Gliwicach. Doświadczenia konstrukcyjne i eksploatacyjne (Equipment C-14 Laboratory in Gliwice. Construction and exploatation experiences). Zeszyty Naukowe Politechniki Ślaskiej, Seria Matematyka-Fizyka, Zesz. 46, Geochronometria 1:55-69.

Ramsey CB. 1995. Radiocarbon calibration and analysis of stratigraphy: the OxCal program. Radiocarbon 37(2):425-30.

Smolarek P. 1991. The underwater investigations of the Polish Maritime Museum in Gdańsk from 1982 to 1985. Acta Universitatis Nicolai Copernici, Archeologia Podwodna 3. Toruń. p 3-24.

Stuiver M, Polach HA. 1977. Reporting of ${ }^{14} \mathrm{C}$ data. $R a-$ diocarbon 19(3):355-63.

Ważny T. 1990. Aufbau und Anwendung der Dendrochronologie für Eichenholz in Polen [PhD thesis]. Hamburg: Buch- und Offsetdruckerei Guenter Stubbemann GmbH. p 213. 